\title{
Effects of N-3 PUFAs Supplementation on Insulin Resistance and Inflammatory Biomarkers in Hemodialysis Patients
}

Z. Rasic-Milutinovic, G. Perunicic, S. Pljesa, Z. Gluvic, S. Sobajic, I. Djuric \& D. Ristic

To cite this article: Z. Rasic-Milutinovic, G. Perunicic, S. Pljesa, Z. Gluvic, S. Sobajic, I. Djuric \& D. Ristic (2007) Effects of N-3 PUFAs Supplementation on Insulin Resistance and Inflammatory Biomarkers in Hemodialysis Patients, Renal Failure, 29:3, 321-329, DOI: $10.1080 / 08860220601184092$

To link to this article: https://doi.org/10.1080/08860220601184092

Published online: 07 Jul 2009.

Submit your article to this journal ¿

Џ Article views: 628

Citing articles: 53 View citing articles 5 


\title{
CLINICAL STUDY
}

\section{Effects of N-3 PUFAs Supplementation on Insulin Resistance and Inflammatory Biomarkers in Hemodialysis Patients}

\author{
Z. Rasic-Milutinovic \\ Department of Endocrinology, University Hospital Zemun/Belgrade, Serbia

\section{G. Perunicic and S. Pljesa} \\ Department of Nephrology and Dialysis, University Hospital Zemun/Belgrade, Serbia
}

Z. Gluvic

Department of Endocrinology, University Hospital Zemun/Belgrade, Serbia

\author{
S. Sobajic and I. Djuric \\ Faculty of Pharmacy, Belgrade, Serbia
}

\section{Ristic}

Institute for Medical Research, Laboratory for Nutrition and Metabolism, Belgrade, Serbia

\begin{abstract}
Aims/Hypothesis. It was suggested that polyunsaturated n-3 fatty acids (n-3 PUFAs) could improve insulin sensitivity and have an anti-inflammatory effects in overall population. This study investigates a possible effect of n-3 PUFAs supplementation on the insulin sensitivity and some inflammatory markers; hence, patients with chronic renal failure (CRF) on maintenance hemodialysis (MHD) are presented with insulin resistance. Methods. This study explored the ratio between red blood cells (RBC) phospholipid long chain fatty acids (LC FAs) and components of metabolic syndrome (MeS) in 35 patients (mean age $54.50 \pm 11.99$ years) with CRF on MHD. Furthermore, the effects of omega-3 FA eight-week's supplementation (EPA+DHA, 2.4g/d) on the MeS features and inflammatory markers TNF-alpha, IL 6, and hsCRP were examined. Results. Supplementation increased EPA and DHA levels in RBCs ( $p=0.009$ for EPA and $p=0.002$ for DHA). Total n-6 PUFAs: n-3 PUFAs ratio tended to be lower after supplementation $(p=0.31)$, but not significantly. Data revealed a significant decrease of saturated FAs (SFA) $(p=0.01)$ as well as total SFA: n-3 PUFAs ratio during the treatment ( $p=$ $0.04)$. The values of serum insulin and calculated IR index-IR HOMA were reduced after supplementation ( $p=0.001$ for both).
\end{abstract}

Address correspondence to Z. Rasic-Milutinovic M.D., Ph.D., Clinical Hospital Zemun/Belgrade, Vukova 9, 11080 Belgrade, Serbia; Tel.: +381-11-3772 715; Fax: +381 112107 057; E-mail: zoricar@eunet.yu
There was a significant decrease in the levels of all inflammatory markers ( $p=0.01$ for TNF alpha, $p=0.001$ for IL $6, p=0.001$ for hsCRP, and $p=0.01$ for ferritin). In multivariate regression analysis, only the changes in n-6 PUFAs: n-3 PUFAs ratio independently contributed to $40 \%$ of the variance in IR HOMA. The impact of changes in PUFAs level in RBCs membrane phospholipid fatty acids on inflammation markers was also registered. The changes in n-6: n-3 PUFAs ratio independently contributed to $18 \%$ of the variance in TNF alpha. Conclusion. It was concluded that the EPA and DHA moderate dose administration in the patients with CRF on MHD had a beneficial effect on insulin resistance decrease. The anti-inflammatory effects of the supplemented PUFAs were also presented.

Keywords end-stage renal disease, insulin resistance, n-6 PUFAs, n-3 PUFAs, inflammatory marker TNFalpha

\section{INTRODUCTION}

Patients with chronic renal failure (CRF) on maintenance hemodialysis (MHD) exhibit plasma fatty acid patterns similar to those with nutritional deficiency of essential fatty acids, ${ }^{[1]}$ as well as limited storage of the $n-3$ fatty acids in adipose tissue, like those in the healthy 
population. ${ }^{[2]}$ This suggestion implied a continued dietary supply. Experimental and in vitro studies have showed that n-3 fatty acids such as -linolenic acid (ALA), fish oil, eicosapentaenoic acid (EPA), and docosahexaenoic acid (DHA) have anti-inflammatory, ${ }^{[3]}$ antithrombotic, ${ }^{[4]}$ and antiarrhythmic properties ${ }^{[5-7]}$ and improve insulin sensitivity. ${ }^{[8]}$ In a prospective study of healthy males, low baseline total n-3 fatty acid, EPA, and DHA levels in the blood are associated with a greater risk of sudden death. ${ }^{[6]}$ In contrast, n-6 fatty acids such as linoleic (LA) and arachidonic acid (AA) have even been reported to promote inflammation, thrombosis, and insulin resistance. ${ }^{[9]}$ Storlien et al. showed that high skeletal muscle membrane n-6 PUFA: n-3 PUFA ratio was inversely related to insulin sensitivity. ${ }^{[10]}$

The high prevalence of insulin resistance and dyslipidemia, the components of metabolic syndrome among CRF patients on $\mathrm{HD},{ }^{[11,12]}$ and a significant negative correlation between calculated IR and the level of ALA in plasma phospholipids were found. ${ }^{[13]}$ Furthermore, significantly lower levels of EPA, DHA, and total n-3 PUFAs in erythrocyte phospholipid fatty acids were found in such patients. ${ }^{[14]}$ In agreement with that finding, the beneficial effects of moderate n-3 PUFA (EPA and DHA) supplementation on the components of metabolic syndrome and atherosclerosis was hypothesized.

\section{SUBJECTS AND METHODS}

\section{Patients}

Forty-two patients were recruited from the HD Unit of Zemun Clinical Hospital. Patients were regularly dialyzed for more than 12 [89 \pm 66$]$ months. Seven patients were dropped out because of intercurrent illness (three patients), dialysis centers change (two patients), and some personal settings (two patients). Exclusion criteria were diabetes mellitus, heart failure (NYHA III or IV), acute myocardial infarction, and acute infectious disorders within three months of the inclusion. Patients were not obese and with no severe malnutrition (BMI $20-30 \mathrm{~kg} / \mathrm{m}^{2}$ ). Patients were dialyzed with synthetic membranes and a bicarbonate dialysate with $1.25,1.5$, or $1.75 \mathrm{mmol} / \mathrm{L}$ calcium according to the serum calcium-phosphate equilibrium and with obligatory use of 1,25-dihydroxy vitamin D3 $\left[1,25(\mathrm{OH})_{2} \mathrm{D} 3\right]$ to control the parathyroid hormone (PTH) levels. The duration of HD was individually tailored (4-6 h weekly) to control body fluids and blood chemistries with the aim to achieve $\mathrm{Kt} / \mathrm{V}>1.2(1.46 \pm$ $0.13)$. Patients did not regularly receive epoetin therapy during that time. Antihypertensives were prescribed when it was necessary to obtain pre-dialysis blood pressure
$<160 / 90 \mathrm{mmHg}$. None of the patients received lipid-lowering drugs, beta-blockers, 1-carnitine, or vitamin $\mathrm{B}_{12}$ three months before the inclusion. Patients regularly took iron and vitamin supplements. Calcium carbonate $\left(\mathrm{CaCO}_{3}\right)$ was used to obtain pre-dialysis serum phosphate level $<2.0 \mathrm{mmol} / \mathrm{L}$. All patients were sedentary $(<1 \mathrm{~h} / \mathrm{wk}$ of physical activity), free of alcohol consumption, and nonsmokers. They maintained their habitual diets $(35 \mathrm{kcal} / \mathrm{kg}$ $\mathrm{BW}$, protein intake $1.0-1.2 \mathrm{~g} / \mathrm{kg} \mathrm{BW}$, fats $<35 \%$ caloric intake) with sodium and potassium restriction. They had low habitual consumption of foods containing soy, fish intake once a week, and no dietary supplementation of oil rich in long-chain fatty acids, as determined by diet assessment made at the time of recruitment. Each patient gave informed written consent to participate in the study, which was approved by the Institutional Ethic Committee.

\section{Study Design}

This study was a cross-sectional and follow-up dietary intervention study. The patients continued to consume high (corn oil-based) n-6 PUFA cooking oils as part of their usual diet, with daily supplementation of n-3 LC PUFA [2.4g EPA+DHA;2:1 EPA/DHA ratio (Natural Wealth Company)] for the eight weeks of the study. N-3 PUFAs were administered in capsules of fish oil supplemented with EPA+DHA (the ratio was 2:1), 2.4g per day, once weekly. The diet control was done as usual for all study participants by interview during HD. The CRF patients were dialyzed three times weekly in the HD unit.

The blood samples were taken to determine the red blood cell phospholipid fatty acids composition before the beginning and at the end of the eight-week n-3 LC-PUFA supplementation period. The fasting blood lipids, glucose, insulin, ionized calcium, serum phosphates, albumin, hemoglobin, iron, ferritin, high sensitivity C-reactive protein (hsCRP), TNF-alpha, and IL-6 were assessed from the same blood samples with a conventional autoanalyzer. Blood samples were taken in the middle of week, after $12-14 \mathrm{~h}$ of overnight fasting and immediately prior to dialysis. Fasting insulin and glucose concentrations were used to calculate insulin resistance from the IR-HOMA model $\left(\right.$ insulin $_{0} \times$ glucose $\left._{0} / 22.5\right) .^{[15]}$

\section{Biochemical Analysis}

Plasma triglyceride, glucose, and total cholesterol concentrations were measured by using standard enzyme color tests (Diagnostics ELITECH, Paris, France); HDL cholesterol was determined by the enzyme procedure, after precipitation by the phosphotungstic acid, in supernatant 
on the Technicon -RA 1000 analyzer; and insulin level was measured using the radioimmunoassay method (INEP Zemun, Belgrade, Serbia). Ferritin was measured by a turbidimetric fixed rate method (Olympus System Reagent; Olympus Diagnostica, Hamburg, Germany). Serum iron was measured by a photometric color test for clinical chemistry analyzers (Olympus System Reagent; Olympus Diagnostica). IL-6 and TNF-alpha concentrations were measured in duplicate by Immunotech IL-6 immunoassays and Immunotech TNF-alpha immunoassays (Beckman Coulter $^{\mathrm{TM}}$, Fullerton, California, USA), and hsCRP was measured by the Olympus (LATEX) assay on the Olympus AU 400 analyzer (Olympus, Pennsylvania, USA). Blood samples for human cytokine determination (human IL- 6 and human-TNF-alpha) were allowed to clot for 30 minutes before centrifugation for 15 minutes at $1000 \mathrm{~g}$, and serum was removed rapidly and carefully from clot. Serum samples were stored in plastic tubes at $-70^{\circ} \mathrm{C}$ until analysis (no longer than one month). Human IL-6 concentrations were measured in duplicate by Immunotech IL-6 immunoassays (IM1120, IM11120) (Beckman Counter ${ }^{\mathrm{TM}}$ ) in serum samples. This ELISA is a one immunological step sandwich-type assay. Samples and standards were incubated in the microtiter plate coated with the first monoclonal antibody anti-IL-6, in the presence of the second anti-IL-6 monoclonal antibody linked to acetylcholinesterase. After incubation, the wells are washed and the bound enzymatic activity is detected by addition of a chromogenic substrate. The intensity of the coloration is proportional to the IL-6 concentration in the sample or standard.

Human TNF-alpha concentrations were measured in duplicate by Immunotech TNF-alpha immunoassays (IM1121, IM11121) (Beckman Counter ${ }^{\mathrm{TM}}$ ) in serum samples. This ELISA test is one-immunological step sandwichtype assay. Samples and standards were incubated in the microtiter plate coated with the first monoclonal antibody anti-TNF-alpha, in the presence of the second anti-TNFalpha monoclonal antibody linked to alkaline phosphatase. After incubation, the wells are washed, and the bound enzymatic activity is detected by addition of a chromogenic substrate. The intensity of the coloration is proportional to the IL- 6 concentration in the sample or standard.

\section{Determination of Red Blood Cell Fatty Acids}

N-6 and n-3 PUFAs content was determined in cell membrane of erythrocytes by gas-liquid chromatography (GC). Total lipids from erythrocyte membranes were extracted, and then free fatty acids separated according to Kates. ${ }^{[16]}$ GC determined the total fatty acid composition in the methyl esters form. Fatty acid methyl esters form were prepared using BF3-methanol solution and extracted with hexane ${ }^{[17]} \mathrm{GC}$ analysis was performed on a VARIAN chromatograph, Model 1400, equipped with a flame ionization detector and a $30 \mathrm{~m} \times 0.32 \mathrm{~cm}$ steel column, packed with LAC-3R-728 (20\%) on Chromosorb W/AW (80-100 mesh). Nitrogen was used as a carrier gas (flow rate $24 \mathrm{~mL} / \mathrm{min}$ ). The $\mathrm{GC}$ oven temperature was kept on $180^{\circ} \mathrm{C}$. The detector and injector temperature was $200^{\circ}$. GC analysis was performed according to the International Standards. Fatty acids were identified by comparison with retention times (Rt) of standards (Supelco TM Fame Mix). Relative content of each fatty acid was calculated from the ratio of the relevant peak area to the total peak area, and the results were expressed as percentage of total identified phospholipid fatty acids.

\section{Statistical Analysis}

Statistical analyses were performed with the SSPS statistical package (SPSS, Chicago, Illinois, USA). Significance was accepted at the level of $p<0.05$. Before statistically analyzed, all data were examined for normality by using the Shapiro-Wilks test. Differences in the absolute changes in fasting plasma triglycerides, total cholesterol, HDL cholesterol, hs-CRP, TNF-alpha, IL-6, glucose, insulin, iron and ferritin concentrations, and calculated IRHOMA over the eight-week period of fish-oil supplementation were determined using independent-sample $t$ tests or Kruskal-Wallis test for variables with skewed distribution. Results are presented as group means ( \pm SDs), or median (interquartiles range). Changes in erythrocyte membrane phospholipid fatty acids composition were assessed by using independent-sample $t$ tests or Kruskal-Wallis test. Variables with a skewed distribution (serum insulin, IR HOMA, inflammatory cytokines, iron, ferritin, and some PUFAs) were naturally log transformed for analyses involving continuous variables. Covariates with missing values were assigned the group mean.

\section{RESULTS}

The baseline characteristics of the 35 MHD patients who completed the study are presented in Table 1 . There were no significant differences in the anthropometrical characteristics of MHD patients before and after eight weeks of fish oil supplementation. A significant decrease of the insulin, calculated IR HOMA, inflammatory marker [TNF-alpha, IL-6, hs-CRP] and ferritin levels $(p<0.01$ for all mentioned variables) were observed after dietary intervention regime. Its influence on fasting plasma triglyceride, total cholesterol, and serum iron levels were not significant $(p>0.05)$. 
Table 1

The baseline characteristics of study participants and responsiveness to fish oil supplementation after eight weeks

\begin{tabular}{lccc}
\hline Variable & Before supplementation & After supplementation & Significance $^{*}$ \\
\hline Age (years) & $54.5 \pm 12$ & & \\
Gender (male/female) & $20 / 15(54.3 \% / 45.7 \%)$ & & 0.93 \\
Hemodialysis duration (months) & $48(24-82)$ & $22.31 \pm 2.86$ & 0.86 \\
Body mass index $\left(\mathrm{kg} / \mathrm{m}^{2}\right)$ & $22.17 \pm 2.99$ & $85.65 \pm 8.40$ & 0.21 \\
Waist $(\mathrm{cm})$ & $86.47 \pm 9.90$ & $2.19 \pm 0.88$ & 0.84 \\
Triglycerides (mmol/L) & $2.30 \pm 0.68$ & $4.90 \pm 1.02$ & 0.08 \\
Total cholesterol (mmol/L) & $5.31 \pm 1.13$ & $1.14 \pm 0.17$ & 0.51 \\
HDL cholesterol $(\mathrm{mmol} / \mathrm{L})$ & $0.91 \pm 0.14$ & $4.94 \pm 0.79$ & $0.001^{\dagger}$ \\
Glucose $(\mathrm{mmol} / \mathrm{L})$ & $5.31 \pm 0.90$ & $17.6(14.40-27.90)$ & $0.001^{\dagger}$ \\
Insulin $(\mathrm{mU} / \mathrm{L})$ & $35.25(26.90-59.20)$ & $3.55(2.77-6.97)$ & $0.001^{\dagger}$ \\
IR HOMA & $8.67(6.01-15.30)$ & $3.7(1.4-10.9)$ & $0.01^{\dagger}$ \\
hs-CRP $(\mathrm{mg} / \mathrm{L})$ & $24.6(19.7-30.5)$ & $0.45(0.23-0.71)$ & $0.001^{\dagger}$ \\
TNFalpha $(\mathrm{pg} / \mathrm{ml})$ & $2.08(0.99-2.82)$ & $0.30(0.17-0.78)$ & $0.23^{\dagger}$ \\
IL-6 $(\mathrm{pg} / \mathrm{ml})$ & $1.60(1.16-8.04)$ & $20.6(9.9-27.0)$ & $0.01^{\dagger}$ \\
Iron $(\mu \mathrm{g} / \mathrm{L})$ & $13.15(6.9-17.9)$ & $310.9(164.5-382.8)$ & \\
Ferritin $(\mu \mathrm{g} / \mathrm{L})$ & $462.0(262.0-807.2)$ & & \\
\hline
\end{tabular}

Values are mean \pm SD or median (interquartile range).

* Independent sample $t$ test or ${ }^{\dagger}$ Kruskal-Wallis test for skewed variables.

\section{Erythrocyte Membrane Phospholipid Fatty Acids Composition before and after Fish-Oil Supplementation}

Fish-oil supplementation over eight weeks resulted in significant changes of some erythrocyte phospholipid fatty acids composition, as presented in Table 2. The significant increase of n-3 PUFAs, ALA EPA, and DHA $(p<0.01)$ was obtained at the end of study period, as well as significant increase of some n-6 PUFAs, LA $(p=0.02)$, gamma linolenic acid, and AA $(p<0.01)$. Furthermore, total n-3 and n-6 PUFAs were not significantly increased after supplementation $(p<0.01)$. Total SFA and SFA: $n-3$ PUFA ratio significantly decreased after supplementation period ( $p<0.05$ ), which was not observed with n-6 PUFAs: $n-3$ PUFAs ratio $(p>0.05)$.

A low level of EPA independently accounted for $34 \%$ of the variance in IR HOMA in the pretreatment period (see Table 3). The levels of baseline hs-CRP inversely correlated with $\mathrm{n}-3$ PUFAs $(\mathrm{r}=-0.39,0.01<p<0.05)$, opposite to the levels of TNF-alpha, which were positively correlated with n-6 PUFAs: n-3 PUFAs ratio before the supplementation $(\mathrm{r}=0.420, p=0.03)$.

The reduced total n-6 PUFAs: n-3 PUFAs ratio correlated with an IR HOMA reduction $(\mathrm{r}=0.369,0.01$ $<p<0.05)$, an increase of HDL cholesterol $(\mathrm{r}=0.392,0.01$ $<p<0.05)$, as well as a decrease of TNF-alpha $(\mathrm{r}=0.420,0.01<p<0.05)$ after the supplementation period. In multivariate regression analysis, only changes in n-6 PUFAs: n-3 PUFAs ratio associated with changes in AA and serum iron contributed to $40 \%$ of the variance in IR HOMA (see Table 4). In addition, significant correlations between the changes in TNF-alpha and n-6 PUFAs: n-3 PUFAs ratio, AA and MUFAs levels $(0.01<$ $p<0.05)$ were registered. In multivariate regression analysis, only changes in n-6 PUFAs: n-3 PUFAs ratio independently contributed to $18 \%$ of the variance in TNF alpha (see Table 5).

\section{DISCUSSION}

N-6 PUFAs are predominant PUFA in all diets. When the diet is supplemented with n-3 PUFAs, the latter partially replace n-6 PUFAs in the vast majority of the cell's membranes (e.g., erythrocytes, platelets, monocytes, lymphocytes, granulocytes, endothelial cells, neuronal cells, colon cells, and hepatic cells). Competition between n-6 PUFAs and n-3 PUFAs occurs in prostaglandin formation. EPA competes with AA for prostaglandin and leukotriene synthesis at cyclooxygenase and lipoxygenase level. 5-lipoxygenase activity and expression are significantly increased in periferal blood mononuclear cells (PBMCs) of ESRD patients on maintenance HD. ${ }^{[18]}$ TacconeGallucci et al. reported dose-dependent inhibition of 5-lipoxygenase activity after three months of supplementation with omega-3 in daily dose of $2.7 \mathrm{~g}$ EPA+DHA, with reduced lipid peroxidation and LTB4 synthesis. ${ }^{[18]}$ 
Table 2

Erythrocyte phospholipid fatty acids composition before and after eight weeks of fish oil supplementation in HD patients

\begin{tabular}{|c|c|c|c|}
\hline Fatty acid (\% of total) & Before supplementation & After supplementation & Significance ${ }^{*}$ \\
\hline 14:0 & $1.18 \pm 0.48$ & $0.56 \pm 0.32$ & 0.007 \\
\hline $15: 0$ & $0.58(0.35-0.62)$ & $0.21(0.10-0.42)$ & $0.001^{\dagger}$ \\
\hline $16: 0$ & $29.93 \pm 3.52$ & $28.30 \pm 3.70$ & 0.04 \\
\hline $18: 0$ & $13.58 \pm 2.03$ & $12.28 \pm 3.85$ & 0.002 \\
\hline $16: 1(n-7)$ & $0.78 \pm 0.36$ & $1.06 \pm 0.75$ & 0.001 \\
\hline $18: 1(n-9)$ & $23.96 \pm 2.76$ & $23.90 \pm 3.55$ & 0.07 \\
\hline $18: 2(n-6)$ & $12.17(9.98-13.16)$ & $15.80(13.80-17.70)$ & $0.02^{\dagger}$ \\
\hline $18: 3(n-3)$ & $0.18 \pm 0.13$ & $0.30 \pm 0.18$ & 0.01 \\
\hline $18: 3(n-6)$ & $0.10(0.0-0.17)$ & $0.19(0.13-0.28)$ & $0.01^{\dagger}$ \\
\hline $20: 3(n-6)$ & $1.91 \pm 0.54$ & $2.58 \pm 1.10$ & 0.03 \\
\hline $20: 4(n-6)$ & $7.44 \pm 1.14$ & $8.18 \pm 2.70$ & 0.001 \\
\hline $20: 5(n-3)$ & $0.21(0.04-0.34)$ & $0.30(0.18-0.60)$ & $0.009^{\dagger}$ \\
\hline $22: 4(n-6)$ & $0.45 \pm 0.10$ & $0.35 \pm 0.17$ & 0.02 \\
\hline $22: 5(n-3)$ & $0.63 \pm 0.42$ & $0.64 \pm 0.51$ & 0.19 \\
\hline $22: 6(n-3)$ & $1.97 \pm 0.69(1.91[1.56-2.27])$ & $2.53 \pm 0.81(2.44[2.0-3.0])$ & $0.37(p=0.002)^{\dagger}$ \\
\hline PUFA (n-6) & $20.28 \pm 3.15$ & $24.65 \pm 3.58$ & 0.29 \\
\hline PUFA (n-3) & $3.07 \pm 0.81$ & $4.00 \pm 0.97$ & 0.23 \\
\hline PUFA (n-6): (n-3) & $7.15 \pm 2.580$ & $6.52 \pm 1.87$ & 0.31 \\
\hline MUFA & $24.74 \pm 2.76$ & $24.96 \pm 3.38$ & 0.07 \\
\hline SFA & $45.33 \pm 3.14$ & $41.47 \pm 4.45$ & 0.01 \\
\hline SFA:n-3 PUFA & $15.21 \pm 4.51$ & $10.79 \pm 3.11$ & 0.04 \\
\hline
\end{tabular}

Values are mean \pm SD or median (interquartile range). Abbreviations: PUFA=polyunsaturated fatty acids, MUFA=mono-unsaturated fatty acids, SFA= saturated fatty acids.

*Independent sample $t$ test or ${ }^{\dagger}$ Kruskal-Wallis test for skewed variables.

\section{Table 3}

Multiple linear regression analysis equations in HD patients (IR HOMA and fatty acids) before fish oil supplementation

\begin{tabular}{lcc}
\hline Variable & Beta & Significance \\
\hline EPA & -0.487 & 0.03 \\
AA & 0.322 & 0.13 \\
\hline
\end{tabular}

Adjusted $\mathrm{R}^{2}=0.34$.

Table 4

Multiple linear regression analysis equations in HD patients (IR HOMA and fatty acids) after fish oil supplementation

\begin{tabular}{lcc}
\hline Variable & Beta & Significance \\
\hline$\Delta$ n-6 PUFA/n-3 PUFA & 0.675 & 0.002 \\
$\Delta$ AA & 0.301 & 0.10 \\
$\Delta$ iron & 0.150 & 0.32 \\
\hline
\end{tabular}

Adjusted $\mathrm{R}^{2}=0.40$.
Table 5

Multiple linear regression analysis equations in HD patients (TNF alpha and fatty acids) after fish oil supplementation

\begin{tabular}{lcc}
\hline Variable & Beta & Significance \\
\hline$\Delta$ n-6 PUFA/ n-3 PUFA & 0.331 & 0.03 \\
$\Delta$ MUFA & 0.263 & 0.16 \\
\hline
\end{tabular}

Adjusted $\mathrm{R}^{2}=0.18$.

In the present study, the supplementation of LC n-3 PUFAs (EPA+DHA) with relatively high dietary intake of n-6 PUFAs is in the concordance with the hypothesis that fatty acid composition of membrane phospolipids was related to insulin resistance. Also, an improvement of insulin resistance was directly related to the reduction of total n-6 PUFAs: n-3 PUFAs ratio. ${ }^{[8,19]}$ There is clear evidence of altered insulin binding and its action as a result of modification of fatty acids in the membrane phospolipids. ${ }^{[10]}$ Min et al. showed that gestational diabetes was associated with AA and DHA reduction and increased palmitic acid in the red cell phosphoglycerides. ${ }^{[20]}$ The biosynthesis of AA and DHA requires delta- 6 and delta-5 
desaturase that are insulin-dependent. However, it is conceivable that their function could be depressed as in Type 2 DM and obesity. ${ }^{[21]}$ Furthermore, increases of AA and DHA levels in HD patients could be a consequence of repaired insulin sensitivity during supplementation period. The present results are in accordance with the study of Pischon et al., ${ }^{[2]}$ who explained that high intake of $n-6$ PUFAs did not antagonize anti-inflammatory effects on n-3 PUFAs. Furthermore, n-6 PUFAs lower the levels of soluble TNF receptors indirectly by reducing insulin resistance. However, many human trials confirmed that high intake of n-3 and n-6 PUFAs could inhibit the activity of the cyclooxygenase and reduce inflammatory mediators. ${ }^{[23]}$ PUFAs may also modulate cytokine production through eicosanoid-independent pathways by influencing membrane composition and fluidity, affecting signal transduction processes or binding to or affecting nuclear receptors such as PPARs or nuclear factor-kB. ${ }^{[24]}$ Observational data suggested that dietary fish oil and ALA are inversely correlated with CRP levels. Caughey et al. demonstrate that a diet enriched with flaxseed oil could inhibit the ex vivo production of inflammatory cytokines IL-1 beta and TNF-alpha by $30 \%$ in four weeks, whereas nine grams of fish oil for another four weeks inhibited IL-1beta by $80 \%$ and TNF-alpha by $74 \% .{ }^{[25]}$ In the study of Endres, IL-1beta, IL-1alpha, and TNF-alpha were decreased for $42 \%$ at six weeks, but a further decrease was observed after 10 weeks after the discontinuation of treatment with fish oil. Therefore, fish oil probably induces suppression of IL-1, contributing to the amelioration of clinical signs and symptoms of disease activity in patients of rheumatoid arthritis to a greater extent than does inhibition of leukotriene metabolism. ${ }^{[26]}$

It was shown in the experimental model that the treatment of diabetic Goto-Kakizaki rats with AA and zinc resulted in insulin resistance improvement. ${ }^{[27]}$ As was published previously, RBCs membrane phospholipid fatty acids composition in HD patients is altered, with significantly lower levels of EPA, DHA, and total n-3 PUFAs comparedto those in control subjects. ${ }^{[14]}$ Friedman et al. recently suggested that HD patients be at risk for inadequate n-3 fatty acids intake and, consequently, its lowered blood levels. ${ }^{[28]}$ It was shown here that low levels of n-3 fatty acids were replaced by higher levels of palmitic and oleic acids. A significant correlation between erythrocyte membrane phospholipid fatty acids composition and calculated IR HOMA was estimated, particularly for the ratio of SFA to n-3 PUFA, EPA, and for AA before supplementation with n-3 PUFAs. However, after eight weeks of supplementation only n-6 PUFAs: n-3 PUFAs ratio independently predicted insulin resistance improvement. According to the study conducted in non-HD patients, the imbalance in dietary n-6 PUFAs: n-3 PUFAs could play a significant role in insulin sensitivity, thereby affecting insulin-sensitive components, such as plasma triacylglycerol. ${ }^{[29]}$ Fiedler et al. carried out the study with lower doses of n-3 PUFAs (1.2g/d) in MHD patients, and levels of triglycerides and very-low-density lipoproteins were decreased after 12 weeks of supplementation; however, the high level of homocystein persisted. The authors supposed that only high doses of omega- 3 fatty acids given for a longer period had influenced inflammation and atherosclerosis. $^{[30]}$

A significant increase in EPA and DHA as well as a significant decrease of SFAs percentage in membrane phospholipids was registered in our HD patients after the eight weeks of supplementation. Also, a significant increase in total n-3 PUFAs and n-6 PUFAs was noted. The n-6 PUFA: n-3 PUFA ratio was not significantly modified, despite its tendency to become lower. The increased levels of the erythrocyte phospholipid's n-6 PUFAs (18:2, 18:3, 20:3, 20:4) after supplementation could be explained via ordinary diet changes (against our recommendations, the patients made an effort to have "healthy" diet with more fruits, whole grains, and vegetables). That probably was the reason of 18:3n-3 (ALA) increase. A lower increase of 22:6n-3 (DHA) is likely to be a consequence of short-term supplementation, and DHA has not been sufficiently incorporated in erythrocyte membrane phospoholipids because less time is needed for its incorporation than for EPA. However, shortened red blood cell life span as one of the renal anemia features, with the mean half-life $22-27$ days, ${ }^{[31]}$ determined the duration of treatment after eight weeks to see the effects.

In this study, a significantly reduced level of SFAs probably prevented deleterious effects of both palmitic acid and elevated blood glucose levels on pancreatic beta-cells function. This is in agreement with the previous reports, in which the formation of ceramide and activation of the apoptotic mitochondrial pathway, which was given up by oleic acid, ${ }^{[32]}$ explained the deleterious effects of palmitic acid. Summers et al. reported that the substitution of dietary SFA with PUFAs improved insulin sensitivity but with changes in distribution of abdominal fat. ${ }^{[33]}$ An increasing body of evidence suggests that supplements of $n-3$ marine FAs may improve defects in insulin signaling and prevent alterations in glucose homeostasis and further development of type 2 diabetes. These effects are likely mediated through the peroxisome proliferator-activated receptors (PPARs), which are up-regulated by polyunsaturated C20 and C22 fatty acids, including EPA and DHA, and in turn are related to the gene expression involved in lipid oxidation and synthesis. ${ }^{[34]}$ Other, pleiotrophic effects of n-3 PUFAs may contribute to decrease the burden of the metabolic syndrome, such as the modulation of inflammation, platelet activation, endothelial function, and blood pressure. ${ }^{[35]}$ 
It was shown here that changes in the n-6 PUFAs: n-3 PUFAs ratio independently contributed to $40 \%$ of the variance in IR HOMA of MHD patients. Increased n-6 PUFAs did not attenuate the effects of long-chain $n-3$ PUFAs on insulin sensitivity, as the n-6 PUFAs: n-3 PUFAs ratio tended to be lower than before supplementation, something Brady et al. showed in healthy subjects. ${ }^{[36]}$ Minor changes of AA in multivariate modeling did not significantly influence on the insulin resistance. Other parameters of metabolic syndrome, such as levels of triglyceride and HDL cholesterol, did not change significantly. However, the change of HDL cholesterol was associated with a decrease of n-6 PUFAs: n-3 PUFAs ratio.

It is well established that patients with CRF on MHD are often malnourished, with the presence of chronic inflammation and higher level of pro-inflammatory biomarkers. ${ }^{[37]}$ Although various factors related to the dialysis procedure may contribute to inflammation in ESRD, a number of non-dialysis-related factors are also important. As only non-overweight subjects were recruited, the impact of obesity on the presence of inflammation was excluded. However, inflammation and protein energy malnutrition are closely related to each other in dialysis patients and appear to play a central role in poor clinical outcome. ${ }^{[38]}$ Berstad et al. have investigated the effect of fish oil supplementation on atherosclerotic activity and found that a large increase in serum EPA and especially DHA might increase inflammation in vascular endothelium through an increase in the level of sVCAM-1. ${ }^{[39]}$ The same authors also noted that there was no significant interaction between the dietary intervention and n-3 PUFAs supplementation. On the other hand, a moderate dietary intake in healthy human volunteers with fish oil-derived n-3 PUFA results in a decrease of monocyte and neutrophil chemotaxis as well as production of proinflammatory cytokines. ${ }^{[40]} \mathrm{N}-3$ PUFA incorporation into an olive oil diet significantly decreased colonic TNF and $\mathrm{LTB}_{4}$ levels compared with colitic rats that were not supplemented with fish. ${ }^{[41]}$ These n-3 PUFAs (EPA and DHA) induce $\mathrm{PGE}_{3}$ and $\mathrm{LTB}_{5}$ production, with lower potency as proinflammatory mediators. ${ }^{[41]}$ The level of TNF-alpha in the colon of rats was almost four-fold lower after supplementation. The findings that plasma FAs composition is linked to inflammatory activity in subjects with insulin resistance ${ }^{[42]}$ suggest possible associations in MHD patients. However, the current study identified only a strong association between changes in n-6 PUFAs: n-3 PUFAs ratio and TNF-alpha levels after supplementation with $\mathrm{n}-3$ PUFAs. As is already known, TNF-alpha impairs insulin signaling by inhibiting the function of IRS-1 through serin phosphorylation, and one could speculate that $n-6$ to $n-3$ PUFAs ratio could also modify insulin resistance indirectly, through TNF-alpha. In addition, reduced ferritin level after supplementation according to the finding that elevated ferritin level in patients with metabolic syndrome and CRF are mainly due to inflammatory mechanisms rather than to iron overload. ${ }^{[36,43]}$ It seems that moderate n-3 LCPUFA supplementation over the eight-week period directly and indirectly affected insulin resistance in HD patients. The ratio n-6 to n-3 PUFAs was crucial for the reduction of insulin resistance in our non-obese and only moderately malnourished MHD patients. Limited positive effects on inflammatory markers-especially on TNF-alpha, which participate in metabolic syndrome as adipokine-were also noticed. One can speculate that a higher dose and longer period of n-3 PUFAs supplementation can promote a higher increase in erythrocyte membrane phospholipid fatty acids, EPA and DHA, and consequently could be suitable for an improvement of metabolic syndrome.

In conclusion, supplementation with n-3 LC PUFAs in HD patients is useful in regard to the reduction of insulin resistance and TNF-alpha levels, which is associated with changes in the n-6: n-3 PUFAs ratio.

\section{ACKNOWLEDGMENTS}

The study received a Hemofarm grant on HD research in 2003. This research was also supported by a grant from the Serbian Ministry of Science and Environmental Protection No. 145071, Serbia and Montenegro. The authors are thankful to all of the patients and healthy volunteers who participated in the study and the Natural Wealth Company for the donation of the fish oil capsules.

\section{REFERENCES}

1. Koorts AM, Viljoen M, Kruger MC, et al. Red blood cell fatty acid profile of chronic renal failure patients receiving maintenance haemodialysis treatment. Prostaglandins Leukot Essent Fatty Acids. 2002; 67:13-18.

2. Arterburn LM, Hall EB, Oken H. Distribution, interconversion, and dose response of $\mathrm{n}-3$ fatty acids in humans. Am J Clin Nutr. 2006;83: S1467-S1476.

3. de Caterina R, Cybulsky MI, Clinton SK, Gimbrone Jr MA, Libby P. The omega-3 fatty acid docosahexaenoate reduces cytokine-induced expression of proatherogenic and proinflammatory proteins in human endothelial cells. Arterioscler Thromb. 1994;14:1829-1836.

4. Sorensen JD, Olsen SF, Pedersen AK, Boris J, Secher NJ, FitzGerald GA. Effects of fish oil supplementation in the third trimester of pregnancy on prostacyclin and thromboxane production. Am J Obstet Gynecol. 1993;168:915-922.

5. Albert CM, Campos H, Stampfer MJ, et al. Blood levels of long chain n-3 fatty acids and the risk of sudden death. NEJM. 2002;346:1113-1118. 
6. Gruppo Italiano per lo Studio della Sopravvivenza nell'Infarto Miocardico. Dietary supplementation with n-3 polyunsaturated fatty acids and vitamin $\mathrm{E}$ after myocardial infarction: results of the GISSI-Prevenzione trial. Lancet. 1999;354:447-455.

7. Leaf A, Kang JX, Xiao YF, et al. Clinical prevention of sudden cardiac death by n-3 polyunsaturated fatty acids and mechanism of prevention of arrhythmias by n-3 fish oils. Circulation. 2003;107:2646-2652.

8. Delarue J, Couet C, Cohen R, et al. Effects of fish oil on metabolic responses to oral fructose and glucose loads in healthy humans. Am J Physiol. 1996;270:E353-E362.

9. Laaksonen D, Nyyssonen K, Niskanen L, Rissanen T, Salonen J. Prediction of cardiovascular mortality in middleaged men by dietary and serum linoleic and polyunsaturated fatty acids. Arch Intern Med. 2005;165:193-199.

10. Storlien LH, Pan DA, Kriketos AD, et al. Skeletal muscle membrane lipids and insulin resistance. Lipids. 1996;31:261S-265S.

11. Rašić Zorica G, Peruničić S, Plješa LJ, Bokan LJ. Insulin resistance and dyslipidemia in hemodialysis patients. Diabetologia. 1997;40(Suppl.1):A421.

12. Rašic-Milutinović Z, Perunicic G, Plješa S, Milić N. Insulin resistance syndrome and other cardiovascular risk factors in hemodialysis patients. Cardionephrology 8, $10^{\text {th }}$ Assisi European Meeting on Cardionephrology, Assisi, April 1-3, 2004, pp. 49-52.

13. Rašić Z, Peruničić G, Plješa S, Ristić V. Serum phospholipids non-esterified very long-chain PUFA and insulin sensitivity in hemodialysis patients. Nephrol Dial Transplant. 2003;18(4):447 (T524).

14. Risitc V, Tepšić V, Ristić-Medic D, Perunicic G, Rasic Z, Poštić M, Arsić A, Blaženčić V, Ristić G. Plasma and erythrocyte phospholipid fatty acids composition in Serbian hemodialysis patients. Renal Failure. 2006; 28:211-216.

15. Matthews DR, Hosker JP, Rudenski AS, Naylor BA, Treacher DF, Turner RC. Homeostasis model assessment. Diabetologia. 1985;28:412-419.

16. Kates M, Techniques of Lipidology, Amsterdam: Elsevier Publ. Co; 1972.

17. Metcalfe LD, Schmitz AA. Annal. Chem. 1961;33:363.

18. Taccacone-Gallucci M, Manca-di-Villahermosa S, Battistini L, Stuffler RG, Tedesco M, Maccarone M. N-3 PUFAs reduce oxidative stress in ESRD patients on maintenance HD by inhibiting 5-lipoxygenase activity. Kidney Int. 2006;69:1450-1454.

19. Borkman M, Storlien LH, Pan DA, Jenkins AB, Chisholm DJ, Campbell LV. The relation between insulin sensitivity and the fatty acid composition of skeletal muscle phospholipids. New Engl J Med. 1993;328:238-244.

20. Min Y, Ghrebremeskel K, Lowy C, Thomas B, Crawford MA. Adverse effect of obesity on red cell membrane arachidonic and docosahexaenoic acids in gestational diabetes. Diabetologia. 2004;47:75-81.

21. Pan DA, Lillioja S, Milner MR, et al. Skeletal muscle membrane lipid composition is related to adiposity and insulin action. J Clin Invest. 1995;96:2802-2808.
22. Pischon T, Hankinson S, Hotamisligil G, Rifai N, Willett W, Rimm E. Habitual dietary intake of n-3 and n-6 fatty acids in relation to inflammatory markers among US men and women. Circulation. 2003;108:155-160.

23. Cho HP, Nakamura M, Clarke SD. Cloning, expression and fatty acids regulation of the human delta-5 desaturase. $J$ Biol Chem. 1999;274:37335-39339.

24. Graber R, Sumida C, Nunez EA. Fatty acids and cell signal transduction. J Lipid Mediat Cell Signal. 1994;9:91-116.

25. Caughey GE, Mantzioris E, Gibson RA, Gleland LG, James MJ. The effect on human tumor necrosis factor alpha and interleukin I beta production of diets enriched in n-3 fatty acids from vegetable oil or fish oil. Am J Clin Nutr. 1996;63:116-122.

26. Endres S, Ghorbam R, Kelley VE, Georgilis K, Lonnemann G, Van der Meer JW, Cannon JG, Rogers TS, Klempner MS, Weber PC, et al. The effect of dietary supplementation with n-3 polyunsaturated fatty acids on the synthesis of interleukin-1 and tumor necrosis factor by mononuclear cells. $N$ Engl J Med. 1989;320:265-271.

27. Song MK, Hwan IK, Rosenthal MJ, et al. Antidiabetic action of arachidonic acid and zinc in genetically diabetogenic Goto-Kakizaki rats. Metabolism. 2003;52:7-12.

28. Friedman AN, Moe SM, Perkins SM, Li Y, Watkins BA. Fish consumption and omega-3 fatty acid status and determinants in long-term hemodialysis. Am $J$ Kidney Dis. 2006;47:1064-1071.

29. Rustan AC, Nenester MS, Drevon CA. Omega-3 and omega6 fatty acids in the insulin resistance syndrome. Lipid and lipoprotein metabolism and atherosclerosis. Ann NY Acad Sci. 1997;827:310-326.

30. Fiedler R, Mall M, Wand C, Osten B. Short term administration of omega-3 fatty acids in hemodialysis patients with balanced lipid metabolism. J Ren Nutr. 2005;15:253-256.

31. Polenakovic M, Sikole A. Is erythropoietin a survival factor for red blood cells? J Am Soc Nephrol. 1996;7:1178-1182.

32. Maedler K, Oberholzer J, Bucher P, Spinas GA, Donath MY. Monounsaturated fatty acids prevent the deleterious effects of palmitate and high glucose on human pancreatic B-cell turnover and function. Diabetes. 2003;52:726-733.

33. Summers LKM, Fielding KA, Bradshaw VA, Ilic V, Baysen C, Clark CM, Moore MN, Frayn KN. Substituting dietary saturated fat with polyunsaturated fat changes abdominal fat distribution and improves insulin sensitivity. Diabetologia. 2002;45:369-377.

34. Forman BM, Chen J, Evans RM. Hypolipidemic drugs, polyunsaturated fatty acids, and eicosanoids are ligands for peroxisome proliferator-activated receptors. Proc Natl Acad Sci. 1997;94:4312-4317.

35. Carpentier Y, Portois L, Malaisse W. n-3 fatty acids and the metabolic syndrome. Am J Clinic Nutrit. 2006;83:S1449S1504.

36. Brady LM, Lovegrove SS, Lesauvage SVM, Gower BA, Minihane AM, Williams CM, Lovegrove JA. Increased n-6 polyunsaturated fatty acids do not attenuate the effects of long-chain $n-3$ polyunsaturated fatty acids on insulin sensitivity or triacylglycerol reduction in Indian Asians. Am J Clin Nutri. 2004;79:983-991. 
37. Kalantar-Zadeh K, Rodrigez RA, Humphreys MH. Association between serum ferritin and measures of inflammation, nutrition and iron in hemodialysis patients. Nephrol Dial Transplant. 2004;19:141-149.

38. Stenvinkel P. Malnutrition and chronic inflammation as risk factors for cardiovascular disease in chronic renal failure. Blood Purif. 2001;19:143-151.

39. Berstad P, Seljeflot I, Veoierod MB, Hjerkinn EM, Arnesen H, Pedersen JI. Supplementation with fish fatty acids and oil affects the association between very long-chain n-3 polyunsaturated fatty acids in serum and non-esterified soluble vascular cell adhesion molecule-1. Clin Sci. 2003;105:13-20.

40. Calder PC. Polyunsaturated fatty acids, inflammation and immunity. Lipids. 2001; 37:1007-1023.
41. Camuesco D, Gálvez J, Nieto A, Comalada M, RodríguezCabezas ME, Concha A, Xaus J, Zarzuelo A. Dietary olive oil supplemented with fish oil, rich in EPA and DHA (n-3) polyunsaturated fatty acids, attenuates colonic inflammation in rats with DSS-induced colitis. J. Nutr. 2005; 135:687-694.

42. Fernandez-Real JM, Broch M, Vendrel J, Ricart V. Insulin resistance, inflammation, and serum fatty acids composition. Diabetes Care. 2003;26:1362-1368.

43. Bugianesi E, Manzini P, D’Antico S, Van E, Longo F, Leone N, Massarenti P, Piga A, Marchesini G, Rizzetto M. Relative contribution of iron burden, HFE mutations, and insulin resistance to fibrosis in nonalcoholic fatty liver. Hepatology. 2004;39:179-187. 
